\title{
Detection Performance of Chaotic Spreading LPI Waveforms
}

\author{
Jin Yu and Yu-Dong Yao
}

\begin{abstract}
Low probability of intercept (LPI) performance of a direct-sequence (DS) spread-spectrum (SS) system with chaotic spreading sequences is investigated in this paper. Several intercept receivers, including energy detectors, synchronous and asynchronous, coherent and noncoherent structures, which are typically used to detect binary DS SS signals, are examined here to detect the presence of chaotic DS SS signals. A simple detection approach using a binary correlating function to detect nonbinary chaotic sequences is proposed. The expressions of detection probabilities of chaotic spreading signals using those intercept receivers are derived. Comparisons between systems using chaotic and binary sequences are given in terms of the LPI performance, and the performance improvement with chaotic spreading sequences is observed.
\end{abstract}

Index Terms-Chaos, low probability of intercept (LPI), spread spectrum (SS).

\section{INTRODUCTION}

$\mathbf{I}^{\mathrm{N}}$ $\mathrm{N}$ A hostile environment, a commercial or military interceptor may try to detect the presence of radio frequency (RF) energy and then to determine the location of its transmitter. Although direct-sequence (DS) spreading signals have generally good low probability of intercept (LPI) performance, there still exist some intercept detectors, which are able to determine the presence of DS spread-spectrum (SS) signals and even extract their carrier frequencies [1], [2]. Energy detectors (radiometers) determine the presence of radio transmissions based on the energy level between signal-plus-noise $\left(H_{1}\right)$ and the noiseonly hypothesis $\left(H_{0}\right)$. This is not an optimal detection method since it overlooks some known information of the signals. Using known features of the signals, such as chip rates and carrier frequencies [3], the problem of detecting weak SS signals has been formulated as a composite likelihood-ratio hypothesis test (an optimum intercept receiver), and explicit solutions for the DS SS systems using binary pseudo-noise (PN) sequences have been presented in [4]. Recently, the signal interception problem is also studied with a self-recovering receiver for DS SS signals with unknown spreading codes and its performance is analyzed and presented in [5]. To improve the security of the DS SS systems, nonbinary and nonperiodic chaotic sequences, which have been investigated in [2] and [6]-[8] are desirable for covert communications because their pseudo-random waveforms can be very useful in disguising signals as noise. Another advantage of using chaotic sequences is the large number of available spreading sequences for multiple-access applications [2].

Manuscript received August 15, 2003; revised December 17, 2003 and January 24, 2004; accepted February 12, 2004. The editor coordinating the review of this paper and approving it for publication is G. Leus.

The authors are with the Wireless Information Systems Engineering Laboratory (WISELAB), Department of Electrical and Computer Engineering, Stevens Institute of Technology, Hoboken, NJ 07030 USA (e-mail: jyu @ stevens.edu; yyao@stevens.edu).

Digital Object Identifier 10.1109/TWC.2004.842948
Binary DS SS signals have been investigated extensively in terms of their LPI performance [1], [3], [4]. The LPI performance bounds for SS systems with error-correcting coding are evaluated in [9]. However, the LPI performance of chaotic signals is only examined considering simple energy detections [2]. In this paper, various intercept receivers, which have been used for the detection of binary DS SS signals, are examined for detecting chaotic DS SS signals, in which the chaotic sequence is unknown and information data are not of interest. A simple binary detection scheme is proposed to detect chaotic sequences, which significantly reduces the complexity of chaotic detectors. This approach is also applicable to any DS SS system with multilevel spreading sequences. We also study the energy detectors in detecting chaotic signals and present the LPI performance. After the introductory section, Section II describes a chaotic system model. The LPI performance of the chaotic signals is analyzed in Section III. Numeric results are shown in Section IV and comparisons with DS SS systems using binary sequences are also presented in this section. Finally, concluding remarks are given in Section V.

\section{ChaOtic System Model}

\section{A. Chaotic Sequences}

In conventional DS SS systems using binary PN sequences, squaring the spread signals or using code clock extraction techniques can wipe out the spreading sequence and leave out the unspread modulated carrier. Thus, the communication is subject to interception by adversary receivers [2]. In order to improve the covertness of the communications, noise-like chaotic spreading sequences can be used to conceal the signals. Chaotic sequences are usually generated using discrete chaotic maps, such as the logistic map, triangular map, and exponential map [6]-[8]. The logistic map is one of the simplest and most widely studied

$$
x_{n+1}=\alpha x_{n}\left(1-x_{n}\right)
$$

where $0 \leq x_{n} \leq 1,0 \leq \alpha \leq 4$, and $\alpha$ is called a bifurcation parameter. Depending on the value of $\alpha$, the dynamics of this system can change dramatically, exhibiting periodicity or chaos. For $0 \leq \alpha<3.57$, the sequence $\left\{x_{n}\right\}$ is periodic with a period $2^{m}$ for some integer $m$; while for $3.57 \leq \alpha \leq 4$, the sequence is, for all practical purposes, nonperiodic and nonconverging [2], [6]-[8]. The histogram of the noise-like chaotic sequences in some cases has been proven to follow a certain probability density distribution (pdf) [2]. For example, the sequences based on the chaotic triangular map are uniform over the interval $[0,1]$. The pdf of the sequences generated from the logistic map takes the following expression:

$$
f\left(x_{n}\right)=\frac{1}{\pi \sqrt{x_{n}\left(1-x_{n}\right)}} .
$$


To change the chaotic sequence range into $[-1,1]$ for bipolar signaling, the following transform is taken, $a_{n}=2 x_{n}-1$. The corresponding pdf of $a_{n}$ is $f\left(a_{n}\right)=\left(1 /\left(\pi \sqrt{\left(1-a_{n}^{2}\right)}\right)\right)$. The sequence $\left\{a_{n}\right\}$ is used as the chaotic spreading sequences throughout this paper.

\section{B. Detection Schemes}

Based on incident waveforms, an intercept receiver must decide between the signal-plus-noise $\left(H_{1}\right)$ and the noise-only hypothesis $\left(H_{0}\right)$

$$
r(t)=\left\{\begin{array}{lll}
\sqrt{2 P} a(t) \cos \left(\omega_{0} t+\phi\right)+n(t), & H_{1} \\
n(t), & H_{0}
\end{array} \quad 0 \leq t \leq T\right.
$$

where $P$ is chosen such that $P E\left[a^{2}(t)\right]$ is the average signal power, $\omega_{0}=2 \pi f_{0}$ is the carrier frequency, $\phi$ is the carrier phase, uniformly distributed in $[0,2 \pi), n(t)$ is additive white Gaussian noise (AWGN) with single-sided spectral density $N_{0} \mathrm{~W} / \mathrm{Hz}, T$ is the observation time, and

$$
a(t)=\sum_{n=-\infty}^{\infty} a_{n} p\left(t-n T_{c}-\epsilon T_{c}\right)
$$

in which $T_{c}$ is the chip period and $p(t)$ is a unit-amplitude pulse of duration $T_{c}$ second. The chip epoch $\epsilon T_{c}$ is modeled by a random variable $\epsilon$, uniformly distributed in $[0,1)$. We assume that a binary correlating function $\left(\operatorname{Sign}\left(a_{n}(t)\right)\right)$ is used to detect a nonbinary chaotic sequence $\left(a_{n}(t)\right)$. This approach is proposed in this paper to simplify the receiver structure and represents a practical implementation approach.

\section{LPI PERFormance of MUltilevel Signals}

The configurations of intercept detectors depend on the amount of known features of the signals. For example, an energy detector only assumes that the signals occupy a bandwidth of $W$ and exist for a time duration $T$. Other intercept receivers could use the known features of the SS signals, such as the carrier frequencies, chip rates, and $T=N T_{c}$, where $N$ is the number of chips in one observation. In the optimum detection for binary sequences, a receiver implements a likelihood ratio test (LRT), which is a procedure based on statistical signal testing of hypotheses [1], [3], [4]. In the following, we evaluate five intercept receivers for chaotic signal detection, considering coherent (known $\phi)$ and noncoherent, synchronous $(\epsilon=0)$ and asynchronous detections, and energy detections.

\section{A. Synchronous Coherent Intercept Receiver}

When synchronous coherent intercept receivers are used to detect the presence of chaotic signals, both the chip epoch $\epsilon T_{c}$ and the carrier phase $\phi$ are assumed to be known, and we have $z(t)=r(t) \cos \left(\omega_{0} t+\phi\right)$ at the receiver side. For binary sequence detection, [3] developed a likelihood ratio test. For nonbinary sequence detection (using binary correlation), following [3], we are able to establish that the decision between $H_{0}$ and $H_{1}$ can be made based on

$$
\Lambda(z(t))=\prod_{j=1}^{N} \exp \left(-\frac{P T_{c}}{N_{0}}\right) \cosh \left(\frac{2 \sqrt{P}}{N_{0}} r_{j}\right) \underset{H_{0}}{\stackrel{H_{1}}{\gtrless}} \Lambda_{0}
$$

where $\Lambda_{0}$ is a threshold and $r_{j}=\int_{(j-1) T_{c}}^{j T_{c}} z(t) d t$. For typical chip signal-to-noise ratio (SNR) below $-5 \mathrm{~dB}$, we have an approximated expression for log-LRT [1]

$$
\lambda=\sum_{j=1}^{N} r_{j}^{2} \underset{H_{0}}{\stackrel{H_{1}}{\gtrless}} \lambda_{0} .
$$

For large values of $N, \lambda$ can be approximated as Gaussian for both noise alone and signal-plus-noise cases and this approximation is quite accurate [1], [3]. Therefore, the detection probability $P_{D}$ can be determined via a $Q$ function. For the random variable $\lambda$, we are able to find its mean and variance

$$
\begin{cases}m_{\lambda}=N\left(N_{0} T_{c}\right)\left(0.5+\gamma_{c} C \delta_{k, 1}\right), & k=0,1 \\ \sigma_{\lambda}^{2}=N\left(N_{0} T_{c}\right)^{2}\left[0.5+\left(2 C \gamma_{c}+D \gamma_{c}^{2}\right) \delta_{k, 1}\right], & k=0,1\end{cases}
$$

where $C=E\left[a_{n}^{2}\right] / E\left[\left|a_{n}\right|\right], D=\operatorname{Var}\left[a_{n}^{2}\right] / E^{2}\left[\left|a_{n}\right|\right], \delta_{k, 1}$ is the Kronecker delta function, which implies the presence of a signal $\left(H_{1}\right)$ for $k=1$ or the absence of the signal $\left(H_{0}\right)$ for $k=0$, and the chip SNR

$$
\gamma_{c}=\frac{P}{N_{0}} \int_{0}^{T_{c}} E\left[a_{n}(t) \operatorname{Sign}\left(a_{n}(t)\right)\right] d t=\frac{P T_{c} E\left[\left|a_{n}\right|\right]}{N_{0}} .
$$

Notice that in deriving or implementing (6), we assume that a binary correlating function $\left(\operatorname{Sign}\left(a_{n}(t)\right)\right)$ is used to detect a nonbinary chaotic sequence $\left(a_{n}(t)\right)$. Therefore, the detectors for binary spreading sequences are applied directly to detect the presence of chaotic spreading signals. This approach is proposed in this paper to simplify the receiver structure. Otherwise, an optimum chaotic receiver structure, which requires the exact correlating functions matching to the received sequences, is not feasible because of the infinite sequence combinations of the nonbinary chaotic signals. In deriving the detection probability, we first find a threshold level $\lambda_{0}$ by setting an acceptable false alarm probability $P_{\mathrm{FA}}$. Using the obtained $\lambda_{0}$, the detection probability $P_{D}$ is derived as a function of $P_{\mathrm{FA}}$

$$
P_{D}=Q\left(\frac{Q^{-1}\left(P_{\mathrm{FA}}\right)-\sqrt{2 N} \gamma_{c} C}{\sqrt{1+4 C \gamma_{c}+2 D \gamma_{c}^{2}}}\right)
$$

where $Q(x)=\int_{x}^{\infty}(1 / \sqrt{2 \pi}) \exp \left(-t^{2} / 2\right) d t$. It is also interesting to note that this binary correlating approach and its related performance analysis are applicable to any DS SS system with multilevel spreading sequences.

\section{B. Synchronous Noncoherent Intercept Receiver}

In this case, the carrier phase $\phi$ is modeled as a random variable with a uniform distribution in $[0,2 \pi)$. While the coherent detection is not possible, [3] and [4] investigated a receiver with matched filters followed by envelope detectors. We use this structure combined with our proposed approach of binary correlating for noncoherent detection of chaotic signals (or multilevel spreading signals). Following [3] and [4] and the derivation steps in Section III-A, we have the same decision rule as (5) with

$$
r_{j}=\sqrt{r_{I_{j}}^{2}+r_{Q_{j}}^{2}}
$$


where

$$
\begin{aligned}
{\left[\begin{array}{l}
r_{I_{j}} \\
r_{Q_{j}}
\end{array}\right] } & =\sqrt{2} \int_{j T_{c}}^{(j+1) T_{c}} r(t)\left[\begin{array}{l}
\cos \omega_{0} t \\
\sin \omega_{0} t
\end{array}\right] d t \\
j & =0, \ldots, N-1 .
\end{aligned}
$$

We further derive the mean and variance of $\lambda$ and obtain

$$
\begin{cases}m_{\lambda}=N\left(N_{0} T_{c}\right)\left(1+\gamma_{c} C \delta_{k, 1}\right), & k=0,1 \\ \sigma_{\lambda}^{2}=N\left(N_{0} T_{c}\right)^{2}\left[1+\left(2 C \gamma_{c}+0.5 D \gamma_{c}^{2}\right) \delta_{k, 1}\right], & k=0,1 .\end{cases}
$$

The detection probability of the chaotic signals is finally derived as

$$
P_{D}=Q\left(\frac{Q^{-1}\left(P_{\mathrm{FA}}\right)-\sqrt{N} \gamma_{c} C}{\sqrt{1+2 C \gamma_{c}+0.5 D \gamma_{c}^{2}}}\right)
$$

Notice that $\gamma_{c}$ in (8) is obtained using the binary decorrelating method.

\section{Asynchronous Coherent Intercept Receiver}

In some practical cases, the chip epoch is unknown and is modeled as a random variable uniformly distributed in $\left[0, T_{c}\right)$. Reference [3] examined this considering two possible epoch values $\left(0,0.5 T_{c}\right)$ and approximated the detection probability averaging over results obtained under the two epoch values. This is equivalent to a two-point quantization of the chip epoch. For chaotic signal (or multilevel spreading signal) detections, in this paper, we first derive a conditional detection probability for a given chip epoch $\epsilon T_{c}$. The final detection probability is obtained through averaging over all possible epoch values. For a given epoch $\epsilon T_{c}$, the Log-LRT decision variable can be found to be

$$
\begin{aligned}
\lambda=\sum_{k=0}^{N-1}\{ & P T_{c}^{2}\left[\epsilon^{2}\left(a_{k}-a_{k+1}\right)^{2}+a_{k+1}^{2}\right. \\
& \left.+2\left(a_{k}-a_{k+1}\right) a_{k+1} \epsilon\right] \\
& \left.+n_{I}^{2}+2 \sqrt{P} n_{I} T_{c}\left(a_{k} \epsilon+a_{k+1}(1-\epsilon)\right)\right\}
\end{aligned}
$$

where $n_{I}=\sqrt{2} \int_{0}^{T_{c}} n(t) \cos 2 \pi f_{0} t d t$. The mean and variance of $\lambda$ are derived as in

$\left\{m_{\lambda}=N\left(N_{0} T_{c}\right)\left(0.5+\gamma_{c} C\left(1-2 \epsilon+2 \epsilon^{2}\right) \delta_{k, 1}\right), \quad k=0,1\right.$ $\left\{\sigma_{\lambda}^{2} \approx N\left(N_{0} T_{c}\right)^{2}\left(0.5+2 C \gamma_{c}\left(1-2 \epsilon+2 \epsilon^{2}\right) \delta_{k, 1}\right), k=0,1\right.$

and the detection probability conditioned on $\epsilon$ is

$$
\begin{aligned}
P_{D \mid \epsilon} & =Q\left(\frac{Q^{-1}\left(P_{\mathrm{FA}}\right)-\sqrt{2 N}\left(1-2 \epsilon+2 \epsilon^{2}\right) \gamma_{c} C}{\sqrt{1+4 C\left(1-2 \epsilon+2 \epsilon^{2}\right) \gamma_{c}}}\right) \\
\epsilon & \in[0,1) .
\end{aligned}
$$

Finally, the detection probability of the signals using an asynchronous coherent receiver is

$$
\overline{P_{D}}=\int_{0}^{1} Q\left(\frac{Q^{-1}\left(P_{\mathrm{FA}}\right)-\sqrt{2 N}\left(1-2 \epsilon+2 \epsilon^{2}\right) \gamma_{c} C}{\sqrt{1+4 C\left(1-2 \epsilon+2 \epsilon^{2}\right) \gamma_{c}}}\right) d \epsilon .
$$

\section{Asynchronous Noncoherent Intercept Receiver}

When both the chip epoch and carrier phase are random variables, following (18) and (19) in [4], the Log-LRT decision variable can be derived as (see Appendix I)

$$
\begin{aligned}
\lambda= & P T_{c}^{2} \sum_{k=0}^{N-1}\left[\epsilon^{2}\left(a_{k}-a_{k+1}\right)^{2}+a_{k+1}^{2}\right]+2 \sqrt{P} T_{c} \epsilon \\
& \times \sum_{k=0}^{N-1}\left(a_{k}-a_{k+1}\right) \\
& \times\left[T_{c} a_{k+1} \sqrt{P}+N\left(n_{I} \cos \phi+n_{Q} \sin \phi\right)\right] \\
& +2 \sqrt{P} T_{c} \sum_{k=0}^{N-1} a_{k+1}\left(n_{I} \cos \phi+n_{Q} \sin \phi\right) \\
& +N\left(n_{I}^{2}+n_{Q}^{2}\right)
\end{aligned}
$$

where $n_{Q}=\sqrt{2} \int_{0}^{T_{c}} n(t) \sin 2 \pi f_{0} t d t$. We further derive the mean and variance of $\lambda$ and have

$\begin{cases}m_{\lambda}=N\left(N_{0} T_{c}\right)\left(1+\gamma_{c} C\left(1-2 \epsilon+2 \epsilon^{2}\right) \delta_{k, 1}\right), & k=0,1 \\ \sigma_{\lambda}^{2} \approx N\left(N_{0} T_{c}\right)^{2}\left(1+2 C \gamma_{c}\left(1-2 \epsilon+2 \epsilon^{2}\right) \delta_{k, 1}\right), & k=0,1 .\end{cases}$

The derivations of $m_{\lambda}$ and $\sigma_{\lambda}^{2}$ are given in Appendix I. The detection probability of the signals conditioned on $\epsilon$ is found as

$$
P_{D \mid \epsilon}=Q\left(\frac{Q^{-1}\left(P_{\mathrm{FA}}\right)-\sqrt{N}\left(1-2 \epsilon+2 \epsilon^{2}\right) \gamma_{c} C}{\sqrt{1+2\left(1-2 \epsilon+2 \epsilon^{2}\right) C \gamma_{c}}}\right)
$$

and the average detection probability of the signals using an asynchronous noncoherent receiver is

$$
\overline{P_{D}}=\int_{0}^{1} Q\left(\frac{Q^{-1}\left(P_{\mathrm{FA}}\right)-\sqrt{N}\left(1-2 \epsilon+2 \epsilon^{2}\right) \gamma_{c} C}{\sqrt{1+2\left(1-2 \epsilon+2 \epsilon^{2}\right) C \gamma_{c}}}\right) d \epsilon .
$$

Note that these detection probabilities [(7), (8), (11), and (14)] are applicable to any DS SS system with multilevel spreading sequences or chaotic sequences.

\section{E. Energy Detector}

The block diagram of an energy detector can be found in [1] and [2]. It consists of a bandpass filter (BPF), a square-law device, a finite-time integrator, a sampler that samples the integrator output at the end of the integration interval $T$, and a threshold comparison device [1]. If the sampler output is greater than the threshold value $K$, a decision is made that signal plus noise was present; if it is smaller than $K$, a decision is made that noise alone was present [1]. The threshold is typically obtained by setting the probability of false alarm. In a chaotic (or multilevel) DS SS system, the output of the integrator of the energy detector is

$$
V=\frac{2}{N_{0}} \int_{0}^{T} r^{2}(t) d t
$$

which can be rewritten as

$$
V=\frac{2}{N_{0}} \sum_{i=0}^{N-1} \int_{i T_{c}}^{(i+1) T_{c}} r^{2}(t) d t .
$$


When the integrator time-bandwidth product $W T$ is large, $V$ is also approximated as Gaussian, and this approximation is quite accurate [1], [3]. The mean and the variance of $V$ are derived in

$$
\begin{cases}m_{V}=2 W T\left(1+\frac{P E\left[a_{n}^{2}\right]}{N_{0} W} \delta_{k, 1}\right), & k=0,1 \\ \sigma_{V}^{2}=4 W T+\left(4 \frac{P^{2} T^{2} \operatorname{Var}\left(a_{n}^{2}\right)}{N_{0}^{2}}+8 \frac{P T E\left[a_{n}^{2}\right]}{N_{0}}\right) \delta_{k, 1}, & k=0,1 .\end{cases}
$$

The derivations of $m_{V}$ and $\sigma_{V}^{2}$ are presented in Appendix II. Using the $Q$-function, the false alarm and detection probability are expressed as

$$
P_{\mathrm{FA}}=Q\left(\frac{K-2 W T}{2 \sqrt{W T}}\right)
$$

and

$$
P_{D}=Q\left(\frac{Q^{-1}\left(P_{\mathrm{FA}}\right)-\sqrt{\frac{N}{2}} \gamma_{c}}{\sqrt{1+\gamma_{c}+\frac{N G \gamma_{c}^{2}}{2}}}\right)
$$

where $\gamma_{c}=P T_{c} E\left[a_{n}^{2}\right] / N_{0}, G=\operatorname{Var}\left[a_{n}^{2}\right] / E^{2}\left[a_{n}^{2}\right], W=$ $2 T_{c}^{-1}$, and $K$ is the threshold value and can be set based on a predetermined false alarm probability, and $\gamma_{c}$ is the predetection average chip SNR. Note that the detection probability of binary PN signals is expressed with $G=0$.

\section{NUMERICAL RESULTS}

Numerical results of the LPI performance for chaotic signals with sequences generated using the logistic map are presented in Figs. 1 and 2. For the chaotic sequences generated from the logistic map (1), we have $G=0.8, C=0.79$, and $D=0.32$, and for those from the triangular map, we obtain $G=0.52$, $C=0.67$, and $D=0.36$. Note that for binary sequences, $G=0, C=1$, and $D=0$. The LPI performance curves of binary PN-based SS signals are also given in Fig. 1 for comparison purposes. From Fig. 1, we observe that chaotic signals have better LPI performance than binary PN signals. The improvement is about $\log _{10}(1 / C)=1 \mathrm{~dB}$ for a logistic map and $1.7 \mathrm{~dB}$ for a triangular map in terms of chip SNR as shown in Fig. 3. Notice that the LPI performance improvement of chaotic signals (the decrease of the detection probability) is due to the binary detection of the nonbinary sequences (i.e., a sequence mismatch). When only energy levels are considered for energy detectors, chaotic signals and binary PN signals are observed to have the same LPI performance as shown in Fig. 1. Fig. 2 compares the LPI performance of chaotic signals with synchronous/asynchronous and coherent/noncoherent detections. It is observed that the synchronous noncoherent detector and the asynchronous coherent detector give similar detection probabilities, while the synchronous coherent detector and the asynchronous noncoherent detector give the highest and lowest detection probabilities, respectively. LPI performance of the signals with different spreading sequences under synchronous

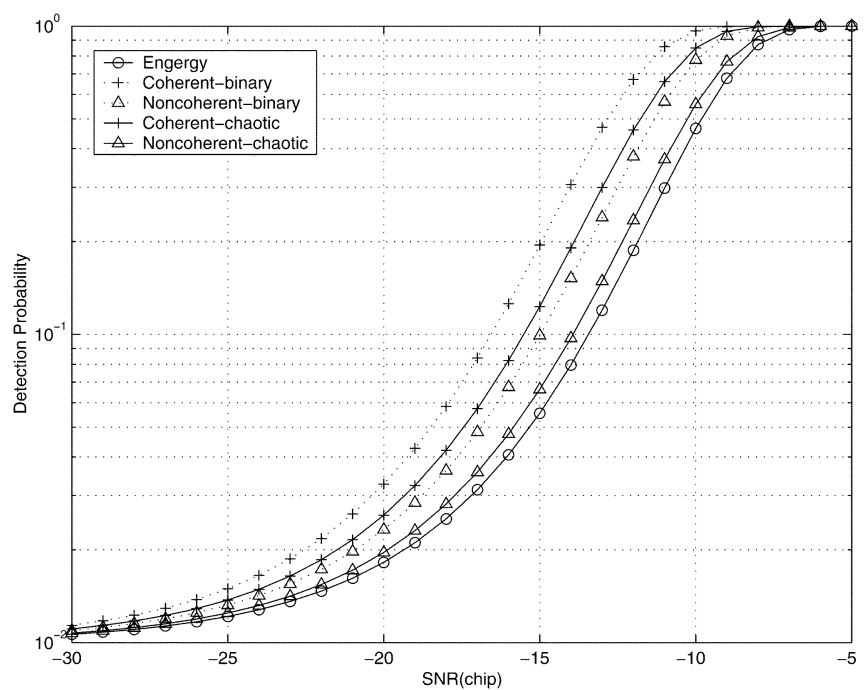

Fig. 1. LPI performances of various intercept receivers with $P_{\mathrm{FA}}=0.01$ and $N=1000$

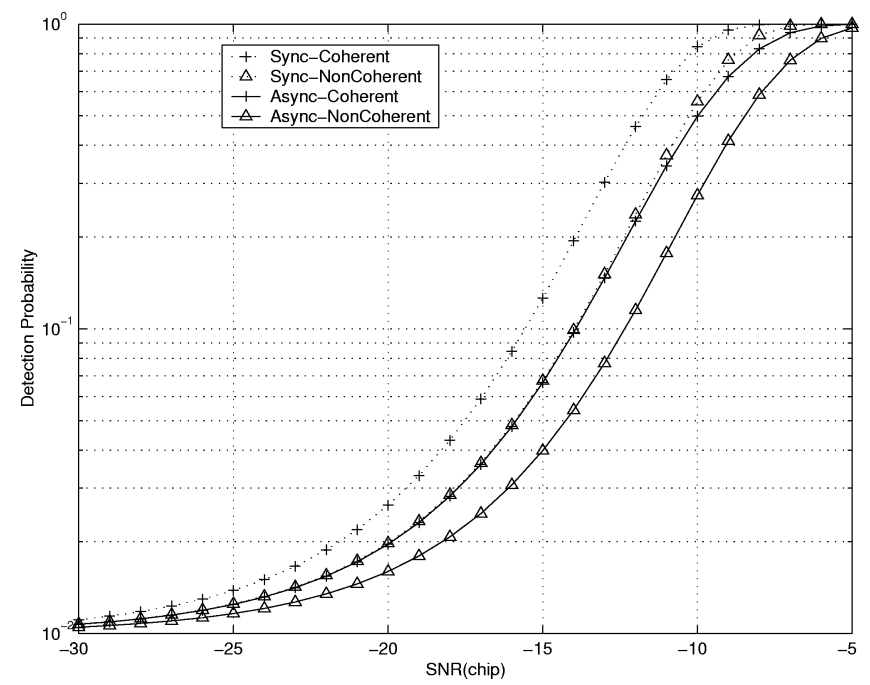

Fig. 2. LPI performances of intercept receivers for synchronous and asynchronous cases with $P_{\mathrm{FA}}=0.01$ and $N=1000$.

coherent detectors are compared in Fig. 3. Simulation results are also shown in this figure and a good agreement with the analytical results is seen.

\section{CONCLUSION}

A simple detection method using a binary correlating function to detect nonbinary chaotic (or multilevel) sequences is proposed in this paper. The chaotic signals are evaluated in terms of LPI performance (detection probabilities) considering synchronous/asynchronous and coherent/noncoherent intercept receivers and energy detectors. Comparisons with binary PN-based SS signals are made and LPI performance advantages of chaotic signals are observed. Notice that binary detection of nonbinary sequences (multilevel or chaotic) leads to nonoptimal detection performance. Optimal detection for multilevel or chaotic sequences will be further investigated. 


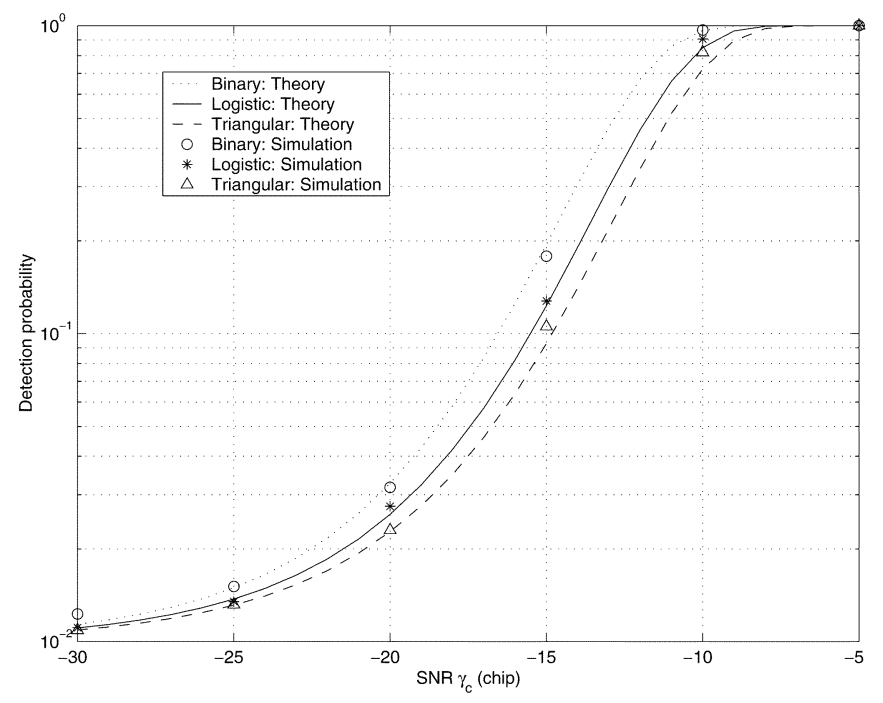

Fig. 3. LPI performances of various sequences under synchronous coherent detectors with $P_{\mathrm{FA}}=0.01$ and $N=1000$, theoretical versus simulation results.

\section{APPENDIX I}

\section{PROOF FOR ASYNCHRONOUS NONCOHERENT INTERCEPT}

\section{RECEIVER}

Instead of approximating the detection probability by averaging over results obtained under the two epoch values $\left(0,0.5 T_{c}\right)$ as in [3], we first derive a conditional detection probability for a given chip epoch $\epsilon T_{c}$ and then the final detection probability is obtained through averaging over all possible epoch values. Considering that binary phase shift keying (BPSK) is used and the carrier phase is independent from one chip to the next, following the derivation in [4] we obtain

$$
\begin{aligned}
\Lambda(Z(t))=k_{1} E_{q, \theta \mid \epsilon} & \times\left\{\operatorname { e x p } \left\{\sum _ { k = 0 } ^ { N - 1 } \operatorname { l n } \left\{\frac{1}{Q} \sum_{q=0}^{Q-1} \frac{1}{2 \pi}\right.\right.\right. \\
& \times \int_{0}^{2 \pi} \exp \left[\frac{2}{N_{0}} \int_{k T_{c}}^{(k+1) T_{c}} r(t) a_{q}^{k}\right. \\
& \left.\left.\left.\left.\times \cos \left(2 \pi f_{0} t+\theta\right) d t\right] d \theta\right\}\right\}\right\}
\end{aligned}
$$

where $k_{1}$ is a constant, $Q=2, a_{0}^{k}=1$, and $a_{1}^{k}=-1$. Thus, we obtain

$$
\begin{aligned}
\Lambda(Z(t))=k_{1} & E_{q, \theta \mid \epsilon}\left\{\operatorname { e x p } \left\{\sum_{k=0}^{N-1} \ln \right.\right. \\
\times & \left.\left.\left\{\frac{1}{Q} \sum_{q=0}^{Q-1} I_{0}\left[\frac{2}{N_{0}}\left[r_{I k}^{2}+r_{Q k}^{2}\right]^{1 / 2}\right]\right\}\right\}\right\}
\end{aligned}
$$

where $I_{0}(x)$ is the zeroth-order modified Bessel function and

$$
r_{I k}=\int_{k T_{c}}^{(k+1) T_{c}} r(t) \cos \left(2 \pi f_{0} t\right) d t
$$

and

$$
r_{Q k}=\int_{k T_{c}}^{(k+1) T_{c}} r(t) \sin \left(2 \pi f_{0} t\right) d t
$$

Then, (20) can be simplified as [4]

$$
\Lambda(Z(t))=k_{1} E_{q, \theta \mid \epsilon}\left\{\exp \left\{\sum_{k=0}^{N-1} \frac{1}{N_{0}^{2}}\left[r_{I k}^{2}+r_{Q k}^{2}\right]\right\}\right\} \text {. }
$$

Let $\tau=\epsilon T_{C}$ and substitute this into $r_{I k}$ and $r_{Q k}$. Scaling $r_{I k}$ and $r_{Q k}$ by $\sqrt{2}$, we obtain

$$
\begin{aligned}
r_{I k}= & \sqrt{2} \int_{k T_{c}-\tau}^{k T_{c}} r(t+\tau) \cos \left(2 \pi f_{0}(t+\tau)\right) d t \\
& +\sqrt{2} \int_{k T_{c}}^{(k+1) T_{c}-\tau} r(t+\tau) \cos \left(2 \pi f_{0}(t+\tau)\right) d t \\
= & \sqrt{P}\left(a_{k} \tau \cos \phi+a_{k+1}\left(T_{c}-\tau\right) \cos \phi\right)+n_{I}
\end{aligned}
$$

and

$$
\begin{aligned}
r_{Q k}= & \sqrt{2} \int_{k T_{c}-\tau}^{k T_{c}} r(t+\tau) \sin \left(2 \pi f_{0}(t+\tau)\right) d t \\
& +\sqrt{2} \int_{k T_{c}}^{(k+1) T_{c}-\tau} r(t+\tau) \sin \left(2 \pi f_{0}(t+\tau)\right) d t \\
= & \sqrt{P}\left(a_{k} \tau \sin \phi+a_{k+1}\left(T_{c}-\tau\right) \sin \phi\right)+n_{Q} .
\end{aligned}
$$

We also have

$$
\begin{aligned}
r_{I k}^{2}= & P \cos ^{2} \phi\left(a_{k}^{2} \tau^{2}+a_{k+1}^{2}\left(T_{c}-\tau\right)^{2}\right. \\
& \left.+2 a_{k} a_{k+1} \tau\left(T_{c}-\tau\right)\right) \\
& +n_{I}^{2}+2 n_{I} \sqrt{P}\left(a_{k} \tau+a_{k+1}\left(T_{c}-\tau\right)\right) \cos \phi
\end{aligned}
$$

and

$$
\begin{aligned}
r_{Q k}^{2}= & P \sin ^{2} \phi\left(a_{k}^{2} \tau^{2}+a_{k+1}^{2}\left(T_{c}-\tau\right)^{2}\right. \\
& \left.+2 a_{k} a_{k+1} \tau\left(T_{c}-\tau\right)\right) \\
& +n_{Q}^{2}+2 n_{Q} \sqrt{P}\left(a_{k} \tau+a_{k+1}\left(T_{c}-\tau\right)\right) \sin \phi .
\end{aligned}
$$

Let $r_{k}^{2}=r_{I k}^{2}+r_{Q k}^{2}$, and we obtain

$$
\begin{aligned}
r_{k}^{2}= & P\left(a_{k} \tau+a_{k+1}\left(T_{c}-\tau\right)\right)^{2}+\left(n_{I}^{2}+n_{Q}^{2}\right) \\
& +2 \sqrt{P}\left(a_{k} \tau+a_{k+1}\left(T_{c}-\tau\right)\right) \\
& \times\left(n_{I} \cos \phi+n_{Q} \sin \phi\right) .
\end{aligned}
$$

Summing $r_{j}, j=0, \ldots, N-1$, and substituting $\tau=\epsilon T_{c}$ into (24), we obtain

$$
\begin{aligned}
\lambda= & \sum_{k=0}^{N-1} r_{k}^{2}=P T_{c}^{2} \sum_{k=0}^{N-1}\left[\epsilon^{2}\left(a_{k}-a_{k+1}\right)^{2}+a_{k+1}^{2}\right] \\
& +2 \sqrt{P} T_{c} \epsilon \sum_{k=0}^{N-1}\left(a_{k}-a_{k+1}\right) \\
& \times\left[T_{c} a_{k+1} \sqrt{P}+\left(n_{I} \cos \phi+n_{Q} \sin \phi\right)\right] \\
& +2 \sqrt{P} T_{c} \sum_{k=0}^{N-1} a_{k+1}\left(n_{I} \cos \phi+n_{Q} \sin \phi\right) \\
& +N\left(n_{I}^{2}+n_{Q}^{2}\right) .
\end{aligned}
$$

Since $\phi$ is a uniform random variable in $[0,2 \pi)$, the mean value of $\lambda$ is

$$
\begin{aligned}
m_{\lambda} & =E[\lambda \mid \epsilon] \\
& =P N T_{c}^{2} E\left[a_{n}^{2}\right]\left(1+2 \epsilon^{2}-2 \epsilon\right)+N E\left[n_{I}^{2}+n_{Q}^{2}\right] \\
& =N\left(N_{0} T_{c}\right)\left(1+\left(1+2 \epsilon^{2}-2 \epsilon\right) \gamma_{c} C\right)
\end{aligned}
$$


and the variance can be found as

$$
\sigma_{\lambda}^{2}=\operatorname{Var}(\lambda \mid \epsilon)=E\left[\lambda^{2} \mid \epsilon\right]-E^{2}[\lambda \mid \epsilon]
$$

where

$$
\begin{aligned}
E\left[\lambda^{2} \mid \epsilon\right]=N E\{ & P^{2}\left(a_{k} \tau+a_{k+1}\left(T_{c}-\tau\right)\right)^{4} \\
& +4 P\left(n_{I}^{2}+n_{Q}^{2}\right)\left(a_{k} \tau+a_{k+1}\left(T_{c}-\tau\right)\right)^{2} \\
& \left.+\left(n_{I}^{2}+n_{Q}^{2}\right)^{2}\right\}
\end{aligned}
$$

Substituting (26) and (28) into (27), we obtain

$$
\begin{aligned}
& \operatorname{Var}(\lambda \mid \epsilon)=N\{ P^{2} \operatorname{Var}\left\{\left(a_{k} \tau+a_{k+1}\left(T_{c}-\tau\right)\right)^{2}\right\} \\
&+2 P N_{0} T_{c} E\left[\left(a_{k} \tau+a_{k+1}\left(T_{c}-\tau\right)\right)^{2}\right] \\
&\left.\quad+\operatorname{Var}\left[n_{I}^{2}+n_{Q}^{2}\right]\right\} \\
& \approx N\left(N_{0} T_{c}\right)^{2}\left(1+2 C \gamma_{c}\left(1-2 \epsilon+2 \epsilon^{2}\right)\right) .
\end{aligned}
$$

\section{APPENDIX II}

\section{PROOF FOR ENERGY DETECTOR}

When signals exist, $r(t)$ can be rewritten as [1]

$$
\begin{aligned}
r(t)=\left(\sqrt{2 P} a(t) \cos \phi+\sqrt{2} n_{I}(t)\right) \cos \left(\omega_{0} t\right) \\
-\left(\sqrt{2 P} a(t) \sin \phi+\sqrt{2} n_{Q}(t)\right) \sin \left(\omega_{0} t\right)
\end{aligned}
$$

where $n_{I}(t)$ and $n_{Q}(t)$ are baseband white Gaussian noise processes with two-sided power spectral densities $N_{0} / 2$ over frequency range $|f|<W / 2$ [1]. Therefore, the mean value of $V$ can be found as in (31), shown at the bottom of the page. Following [1] and [10], we have

$$
\sigma_{V}^{2}=\frac{8 T}{N_{0}^{2}} \int_{0}^{T}\left(1-\frac{x}{T}\right)\left(R_{w}(x)-E^{2}[w(x)]\right) d x .
$$

Assuming that the chaotic chips are independent of each other, we obtain

$$
\begin{aligned}
\sigma_{V}^{2}=\frac{8 T}{N_{0}^{2}} \sum_{n=0}^{N-1} \int_{n T_{c}}^{(n+1) T_{c}} & \left(1-\frac{x}{T}\right) \\
& \times\left(R_{w}(x)-E^{2}\left[w_{n}(x)\right]\right) d x
\end{aligned}
$$

where

$$
\begin{aligned}
w_{n}(x)=P a_{n}^{2}+2 \sqrt{P} & a_{n} \cos \phi n_{I}(x) \\
& +2 \sqrt{P} a_{n} \sin \phi n_{Q}(x)+n_{I}^{2}(x)+n_{Q}^{2}(x)
\end{aligned}
$$

which gives

$$
E\left[w_{n}(x)\right]=P E\left[a_{n}^{2}\right]+N_{0} W
$$

The autocorrelation function is

$$
\begin{aligned}
& R_{w}(x)=E\left[w_{n}(t) w_{n}(t+x)\right] \\
& =E\left\{P^{2} a_{n}^{4}+2 P a_{n}^{2}\left(n_{I}^{2}(t)+n_{Q}^{2}(t)\right.\right. \\
& \left.+n_{I}^{2}(t+x)+n_{Q}^{2}(t+x)\right) \\
& +2 P a_{n}^{2} n_{I}(t) n_{I}(t+x) \\
& +2 P a_{n}^{2} n_{Q}(t) n_{Q}(t+x)+\left(n_{I}^{2}(t)+n_{Q}^{2}(t)\right) \\
& \left.\times\left(n_{I}^{2}(t+x)+n_{Q}^{2}(t+x)\right)\right\} \\
& =P^{2} E\left[a_{n}^{4}\right]+2 P E\left[a_{n}^{2}\right] N_{0} W+4 P E\left[a_{n}^{2}\right] R_{n}(x) \\
& +2 R_{n^{2}}(x)+\frac{\left(N_{0} W\right)^{2}}{2}
\end{aligned}
$$

where

$$
R_{n^{2}}(x)=2 R_{n}^{2}(x)+\frac{\left(N_{0} W\right)^{2}}{4}
$$

and

$$
R_{n}(x)=0.5 N_{0} W \operatorname{sinc}(W x)
$$

Substituting (34)-(37) into (33), we have

$$
\begin{aligned}
\sigma_{V}^{2}= & \frac{8 T}{N_{0}^{2}} \sum_{n=0}^{N-1} \int_{n T_{c}}^{(n+1) T_{c}}\left(1-\frac{x}{T}\right) \\
& \times\left\{4 P E\left[a_{n}^{2}\right] R_{n}(x)+4 R_{n}^{2}(x)+P^{2} \operatorname{Var}\left(a_{n}^{2}\right)\right\} d x \\
= & \frac{16 W T}{N_{0}^{2}} \int_{0}^{T}\left(1-\frac{x}{T}\right) \\
& \times\left\{P E\left[a_{n}^{2}\right] N_{0} W \operatorname{sinc}(W x)\right. \\
& \left.\quad+0.5 N_{0}^{2} W^{2} \operatorname{sinc}^{2}(W x)+0.5 P^{2} \operatorname{Var}\left(a_{n}^{2}\right)\right\} d x
\end{aligned}
$$

For a large value of $W T$, (38) can be simplified as

$$
\sigma_{V}^{2}=\frac{8 T}{N_{0}}\left(P E\left[a_{n}^{2}\right]+0.5 N_{0} W\right)+4 \frac{P^{2} T^{2} \operatorname{Var}\left(a_{n}^{2}\right)}{N_{0}^{2}}
$$

\section{ACKNOWLEDGMENT}

The authors wish to thank the anonymous reviewers for their valuable comments.

$$
\begin{aligned}
m_{V} & =\frac{2}{N_{0}} \sum_{n=0}^{N-1} \int_{n T_{c}}^{(n+1) T_{c}}\left[\left(\sqrt{2 P} a_{n} \cos \phi+\sqrt{2} n_{I}(t)\right) \cos \left(\omega_{0} t\right)-\left(\sqrt{2 P} a_{n} \sin \phi+\sqrt{2} n_{Q}(t)\right) \sin \left(\omega_{0} t\right)\right]^{2} d t \\
& =\frac{2}{N_{0}} \sum_{n=0}^{N-1}\left\{P E\left[a_{n}^{2}\right] T_{c}+\int_{n T_{c}}^{(n+1) T_{c}} E\left[n_{I}^{2}(t)+n_{Q}^{2}(t)\right] d t\right\}=2 W T\left(1+\frac{P E\left[a_{n}^{2}\right]}{N_{0} W}\right)
\end{aligned}
$$




\section{REFERENCES}

[1] R. L. Peterson, R. E. Ziemer, and D. E. Borth, Introduction to Spread Spectrum Communications. Englewood Cliffs, NJ: Prentice Hall, 1995.

[2] G. Heidari-Bateni, "Chaotic signals for digital communications," Ph.D. dissertation, Purdue Univ., Lafayette, IN, 1992.

[3] A. Polydoros and C. L. Weber, "Detection performance considerations for direct-sequence and time-hopping LPI waveforms," IEEE J. Sel. Areas Commun., vol. 3, no. 5, pp. 727-744, Sep. 1985.

[4] N. F. Krasner, "Optimal detection of digitally modulated signals," IEEE Trans. Commun., vol. COM-30, no. 5, pp. 885-895, May 1982.

[5] M. K. Tsatsanis and G. B. Giannakis, "Blind estimation of direct sequence spread spectrum signals in multipath," IEEE Trans. Signal Process., vol. 45, no. 5, pp. 1241-1252, May 1997.
[6] G. Heidari-Bateni and C. D. McGillem, "A chaotic direct-sequence spread-spectrum communication system," IEEE Trans. Commun., vol. 42, no. 1, pp. 1524-1527, Feb./Mar./Apr. 1994.

[7] F. C. M. Lau, M. M. Yip, C. K. Tse, and S. F. Hau, "A multiple-Access technique for differential chaos-shift keying," IEEE Trans. Circuit Syst. I, vol. 49, no. 1, pp. 96-104, Jan. 2002.

[8] G. Mazzini, G. Setti, and R. Rovatti, "Chaotic complex spreading sequences for asynchronous DS-CDMA-Part I: System modeling and results," IEEE Trans. Circuit Syst. I, vol. 44, no. 10, pp. 937-947, Oct. 1997.

[9] E. Chandler and G. Cooper, "Low probability of intercept performance bounds for spread-spectrum systems," IEEE J. Sel. Areas Commun., vol. 3, no. 5, pp. 706-713, Sep. 1985.

[10] A. Papoulis, Probability, Random Variables, and Stochastic Processes, 2nd ed. New York: McGraw-Hill, 1991. 